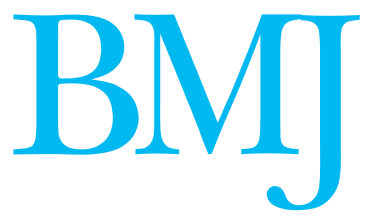

\title{
Diagnosis of coeliac disease
}

\author{
Follow up and review are needed when test results are not clear
}

Clinical review pp 773,775
$\mathrm{T}$ wo articles in this issue remind us about coeliac disease and the need to consider it as a diagnosis in seemingly unusual circumstances-in elderly patients and in obese patients $\left(p\right.$ 773, 775). ${ }^{12}$ Furthermore, in the patient reported by Saunders et al, the usual diagnostic features of anti-endomysial and anti-transglutaminase antibodies and histological enteropathy were absent initially. ${ }^{1}$

Coeliac disease is a relatively common condition. Its prevalence is approximately $0.3-1 \%$ of the population in almost all countries and ethnic groups where it has been investigated. ${ }^{3}$ Previously regarded largely as a childhood problem it is now recognised to affect mostly adults, with about $25 \%$ of patients receiving their diagnosis at over 60 years of age. ${ }^{4}$ When present, the features of malabsorption (diarrhoea and weight loss) should point to the diagnosis, but now a wide range of clinical manifestations are recognised. Patients often have few or no gastrointestinal symptoms and can even be obese. ${ }^{25}$

Conventionally diagnosis is based on the histological finding of villous atrophy in the small bowel, which recovers on a gluten-free diet. Since antibody testing has become available, anti-endomysial and antitransglutaminase antibodies are often used as a preliminary non-invasive means to screen patients and populations.

Where histological examination of the small bowel shows villous atrophy and antibodies are found the diagnosis is straightforward. Difficulties arise when one or other of these is not found, and the diagnosis is particularly difficult when both are negative. ${ }^{1}$ The objective of diagnosis is to determine treatment, which for coeliac disease is a gluten-free diet. Some patients with coeliac disease have no symptoms and find the limitations imposed by a gluten-free diet difficult to accept. When the diagnosis is in doubt and patients have few or no symptoms, deciding about treatment is more problematic.

Where antibody tests are positive but histological findings are normal, the sensitivity and specificity of antibody testing and the reliability of histological interpretation are called into question. Is the antibody test a false positive or the histological examination a false negative? The results of antibody testing in any locale depend on the population used to standardise the test, the particular test used, and the laboratory expertise. In our experience a combination of anti-endomysial and anti-transglutaminase antibodies is highly predictive of the condition $(>95 \%)$. We have also seen transiently positive antibody tests, particularly anti-gliadin ${ }^{6}$ and anti-transglutaminase antibodies, that become negative on retesting and were clearly false positives. By contrast, reports exist of positive antibody testing that predict the development of enteropathy after several years of follow up-for anti-reticulin and anti-gliadin antibodies $^{7}$ and anti-endomysial antibodies (C Feighery, personal communication, 2005).

Histological assessment of small intestinal biopsies needs expertise. Enteropathy at the mild end of the spectrum-with infiltration by inflammatory cells without villous atrophy (Marsh 1 and 2 lesions)-needs to be recognised. ${ }^{8}$ Another pitfall is the way in which small intestinal biopsies are prepared. Specimens need to be correctly oriented so that villi are cut longitudinally. If the mucosa is cut tangentially atrophic villi may look normal and normal villi atrophic. The disease may be patchy, so biopsies from several sites (usually four) in the duodenum are recommended. Although coeliac disease is regarded as a proximal small intestinal disorder, it is possible for it to be more manifest distally, and may require enteroscopy for diagnosis. ${ }^{9}$ Wireless capsule endoscopy may also prove useful for this purpose. Sometimes patients come to the outpatient clinic having started a gluten-free diet after a positive antibody test. If the clinical response has been equivocal and histological findings normal a gluten challenge may help make a diagnosis. This, however, needs further gastroscopy, and uncertainty prevails about when to repeat the biopsy.

What then should be the recommendation for patients with normal histology and a positive test for anti-endomysial or anti-transglutaminase antibodies? The first action should be to review the histology. If the histology shows Marsh 1 or 2 changes and the patient has symptoms treatment could be started. If the histology is normal and the patient has symptoms the options are to treat, to investigate further (for example, with capsule endoscopy or enteroscopy), to review long term, or if the patient has been on a gluten-free diet to review after gluten challenge. If the histology is normal and the patient has no symptoms the options are to review long term or investigate further.

What about patients whose histology is abnormal but who do not have the diagnostic antibodies? The histological appearance of coeliac disease is nonspecific and can result from other conditions such as gastroenteritis. Such changes may persist for some time. However, antibody negative cases of coeliac 
disease do occur, ${ }^{10}$ and if the condition is suspected clinically, biopsy should still be carried out. A possible reason for negative antibody testing is IgA deficiency. This is more common in coeliac disease and since antiendomysial and anti-transglutaminase antibodies are normally measured as immunoglobulin A antibodies they will be absent in patients with $\operatorname{IgA}$ deficiency. Therefore in patients with IgA deficiency, IgG anti-endomysial and anti-transglutaminase antibodies need to be checked.

Successful diagnosis of coeliac disease depends on a high index of suspicion, careful evaluation of investigations, and, where these are not clear, a willingness to review patients and make the diagnosis later in light of clinical progress and results of re-investigation.

\section{R G Peter Watson senior lecturer in medicine}

Department of Medicine, Institute of Clinical Science, Royal Victoria Hospital, Belfast BT12 6BA

(p.watson@qub.ac.uk)

Competing interests: None declared.
1 Saunders DS, Hurlstone DP, McAlindon ME, Hadjivassiliou M, Cross SS, Wild G, et al. Antibody negative coeliac disease presenting with coeliac crisis in the elderly people-an easily missed diagnosis. BMJ 2005;330:775-6

2 Furse RM, Mee AS. Atypical presentation of coeliac disease. BMJ 2005;330:773-4

3 Holmes G, Catassi C. Epidemiology. In: Holmes G, Catassi C. Coeliac disease. Oxford: Health Press, 2000:9.

4 Holmes G, Catassi C. Clinical manifestations. In: Holmes G, Catassi C. Coeliac disease. Oxford: Health Press; 2000:32-3.

5 Watson RGP, McMillan SA, Dickey W, Biggart JD, Porter KG. Detection of undiagnosed coeliac disease with atypical features using antireticulin and and antigliadin antibodies. QJ Med 1992;84:713-8.

6 Johnston SD, Watson RGP, McMillan SA, Evans A, Love AHG. Serological markers for coeliac disease: changes with time and relation to enteropathy. Eur J Gastroenterol Hepatol 1998;10:259-64.

7 Collin P, Helin H, Maki M, Halstrom O, Karvonen AL. Small bowel-mucosal inflammation in reticulin or gliadin antibody-positive patients without villous atrophy. Scand J Gastroenterol 1993;28:595-8.

8 Marsh MN. Gluten, major histocompatibility complex, and the small intestine. A molecular and immunologic approach to the spectrum of gluten sensitivity (celiac sprue). Gastroenterology 1992;102:330-54.

9 Horoldt BS, McAlindon ME, Stephenson TJ, Hadjivasssilou M, Sanders DS. Making the diagnosis of coeliac disease: is there a role for push enteroscopy? Eur J Gastroenterol Hepatol 2004;16:1143-6.

10 Dickey W, Hughes DF, McMillan SA. Reliance on serum endomysial antibody testing underestimates the true prevalence of coeliac disease by one fifth. Scan J Gastroenterol 2000;35:181-3.

\title{
What makes a good clinical decision support system
}

\author{
We have some answers, but implementing good decision support is still hard
}

$\mathrm{C}$ linical decision support is the provision of "clinical knowledge and patient-related information, intelligently filtered or presented at appropriate times, to enhance patient care."1 Medical institutions are increasingly adopting tools that offer decision support to improve patient outcomes and reduce errors. Healthcare providers and administrators with little or no training in computer science may be asked to evaluate, select, or contribute to the development of decision support systems for their practices. Is there an easy way to determine which clinical decision support systems are good?

In this issue Kawamoto and colleagues provide some evidence based guidance in a systematic analysis of the ability of decision support systems to improve practice in both statistically significant and clinically meaningful ways (p 765). ${ }^{2}$ This rigorous review includes only randomised controlled trials and excludes small studies that do not meet $50 \%$ of established criteria for methodological quality. ${ }^{3}$ It identifies four independent predictors of effective decision support: systems that enhance practice generate decision support automatically as part of the normal clinical workflow and at the time and place of decision making; they use computers to deliver support; and they offer specific recommendations rather than mere assessments. Ninety four per cent of clinical decision support systems with these characteristics improved practice compared with only $46 \%$ of systems that lack one of these features.

Similar findings were reported in a recent systematic review of controlled trials evaluating computerised decision support programs, but worry- ing deficiencies in the evidence base were noted. ${ }^{5}$ Garg and colleagues found that the performance of healthcare practitioners using decision support systems improved in $64 \%$ of studies, comparable to the improvement in $68 \%$ of trials noted by Kawamoto et $\mathrm{al}^{2}{ }^{2}$ and they also observed that automatically generated versus user-initiated decision support resulted in better delivery of care. However, of the 100 studies analysed, few specified a primary outcome for statistical analysis, and nearly three quarters were evaluated by their software developers. Developer selfassessment was the only other factor associated with better performance. The outcomes of most studies were metrics assessing the process of healthcare delivery with and without decision support systems. Only 52 trials measured at least one patient outcome, and improvements were noted in only $13 \%$ of these studies.

Unfortunately, the implementation of effective clinical decision support is a challenging task involving interactions between technologies and organisations, and there are no easy solutions to guarantee success or to avoid failure in this complex process. ${ }^{6}$ Many factors influence reductions in errors or improvements in health, so measuring the effectiveness of decision support systems in improving these endpoints is difficult. Moreover, another recent eye opening observational study identified 22 different ways in which an established computerised order entry system (the benefits of which are thought to include reducing errors) could actually introduce medication errors. ${ }^{7}$ Although many researchers have sought to prove the advantages of clinical decision
Information in Practice $\mathrm{p} 765$ 